J. Appl. Numer. Optim. 3 (2021), No. 1, pp. 215-229

Available online at http://jano.biemdas.com

https://doi.org/10.23952/jano.3.2021.1.13

\title{
CONVERGENCE OF RELAXED INERTIAL METHODS FOR EQUILIBRIUM PROBLEMS
}

\author{
DANG VAN HIEU ${ }^{1, *}$, HOANG NGOC DUONG ${ }^{2}$, BUU HUU THAI ${ }^{2}$ \\ ${ }^{1}$ TIMAS - Thang Long University, Hanoi, Vietnam \\ ${ }^{2}$ Department of Basic Sciences, College of Air Force, Nha Trang, Vietnam
}

\begin{abstract}
In this paper, we first introduce a relaxed inertial algorithm for solving a pseudomonotone equilibrium problem with a Lipschitz-type condition in a Hilbert space. The algorithm is constructed around the proximal-like mapping and the inertial technique. The weak convergence of the algorithm is proved under some mild conditions. We also present a modified version of the first algorithm which can be implemented more easily without the prior knowledge of the Lipschitz-type constant of bifunction. Finally, several experiments are performed to illustrate the numerical behavior of the new algorithms, and also to show their computational efficiency over others.
\end{abstract}

Keywords. Extragradient method; Equilibrium problem; Monotone bifunction; Lipschitz-type condition.

\section{INTRODUCTION}

The equilibrium problem (EP) [1, 2], which is well known as the Ky Fan inequality, were early studied in [3]. This problem unifies in a simple form various problems, which arise in economics, optimization and operators research. More precisely, in this general formulation, the equilibrium problem includes an important class of variational inequalities as well as the classes of complementarity problems, convex optimisation, saddle point problems, fixed point problems and famous Nash equilibria problem. In recent years, the equilibrium problem has been intensively and widely investigated both in theoretically and algorithmically. Many numerical methods have been proposed for approximating solutions of this problem; see, e.g., [4]-[16] and the references therein.

One of the most popular methods for solving the equilibrium problem is the proximal point method (PPM). This method was first introduced by Martinet [17] for monotone variational inequality problems. Later, it was extended by Rockafellar [18] to monotone operators. Moudafi [14] and Konnov [19] further extended the PPM to the equilibrium problem with monotone and weakly monotone bifunctions, respectively. The PPM is constructed around the resolvent of a bifunction. Based on this method, many works have been devoted to presenting numerical

\footnotetext{
${ }^{*}$ Corresponding author.

E-mail addresses: dv.hieu83@gmail.com (D.V. Hieu),hn.duongkq85@gmail.com (H.N. Duong), bh.thai83@ gmail.com (B.H. Thai).

Received April 7, 2020; Accepted August 11, 2020.
}

(C)2021 Journal of Applied and Numerical Optimization 
approaches for finding an approximation solution of the equilibrium problem in various ways with different types of conditions.

In this paper, we are first interested in a method of inertial-type. This method originates from heavy ball method (an implicit discretization) of the second-order dynamical systems in time $[20,21,22]$. The main feature of which is that the construction of the next iterate is based on at least the previous two iterates. Recently, inertial-type algorithms have been applied to numerous kinds of problems; see, e.g., [23, 24, 25, 26, 27, 28, 29, 30, 31, 32]. In this direction, by using the resolvent of a bifunction, Moudafi [33] proposed a new inertial method, so-called the second-order differential proximal method, which combines the (relaxed) original PPM with inertial effect. Under certain suitable conditions, Moudafi established the weak convergence of the algorithm. Recently, Chbani and Riahi [34] developed the relaxed inertial proximal point methods in incorporating with the Mann iteration or the Halpern iteration and obtained associated convergence results. Several other recent methods in this direction can be found in $[35,36]$.

We next focus on the method which is based on the auxiliary problem principle, namely, the proximal-like algorithm [4]. This method requires at each iteration to compute two-proximal mappings which is equivalent to solve two strongly convex optimization programs. The proximallike algorithm [4] is also called the extragradient method [15] due to the early obtained results in [37] on saddle point problems. The extragradient method was investigated and further extended the convergence in [15] under the hypotheses of the pseudomonotonicity and the Lipschitz-type condition of bifunctions. In recent years, the extragradient method has received a lot of attention due to its importance in numerical computations. We remark here that the extragradient methods seem to be easier to solve numerically by optimization tools than the PPM [5, 6, 7, 8, 16]. A reason to explain this remark may be due to the fact that it is not easy to compute the resolvent of a bifunction.

In this paper, motivated and inspired by the results in $[33,34,35,36,38]$, we first introduce a new algorithm with inertial form for solving a pseudomonotone equilibrium problem with a Lipschitz-type condition in a Hilbert space. The algorithm uses a relaxed version of the extragradient method to incorporate with inertial terms. The chosen stepsizes in the first algorithm depend on the Lipschitz-type constants of bifunctions. The theorem of convergence is established under certain mild conditions. In the inverse case when the information of the Lipschitz-type constants of bifunctions is unknown, we propose the second algorithm which can be performed more easily. The stepsizes in the second algorithm are updated at each iteration by a cheap computation based on the previous iterates. In order to show the efficiency of the new algorithms, several numerical experiments in comparison with others are also implemented.

The remainder of this paper is organized as follows: Section 2 recalls some definitions and preliminary results used in the paper. Sections 3 and 4 deal with the description of the algorithms and the analysis of their convergence. Finally, in Section 5, we perform several experiments to show the numerical behavior of the new algorithms and also to compare them with others. 


\section{PRELIMINARIES}

Let $H$ be a real Hilbert space and let $C$ be a nonempty closed convex subset of $H$. Let $f$ be a bifunction from $H \times H$ to the set of real numbers $\Re$ such that $f(x, x)=0$ for all $x \in C$. The equilibrium problem (EP) for the bifunction $f$ on $C$ is to find $x^{*} \in C$ such that

$$
f\left(x^{*}, y\right) \geq 0, \forall y \in C \text {. }
$$

Let us denote by $\operatorname{EP}(f, C)$ the solution set of problem (EP). It was well known that solution methods are often relative to some concepts of the monotonicity of a bifunction. Recall that a bifunction $f: H \times H \rightarrow \Re$ is said to be:

(i) strongly monotone on $C$ if there exists a constant $\gamma>0$ such that

$$
f(x, y)+f(y, x) \leq-\gamma\|x-y\|^{2}, \forall x, y \in C ;
$$

(ii) monotone on $C$ if

$$
f(x, y)+f(y, x) \leq 0, \forall x, y \in C ;
$$

(iii) pseudomonotone on $C$ if

$$
f(x, y) \geq 0 \Longrightarrow f(y, x) \leq 0, \forall x, y \in C ;
$$

(iv) strongly pseudomonotone on $C$ if there exists a constant $\gamma>0$ such that

$$
f(x, y) \geq 0 \Longrightarrow f(y, x) \leq-\gamma\|x-y\|^{2}, \forall x, y \in C .
$$

From the aforementioned definitions, it is easy to see that

$$
\text { (i) } \Longrightarrow(\text { ii) } \Longrightarrow \text { (iii) and (i) } \Longrightarrow(\text { iv) } \Longrightarrow \text { (iii). }
$$

A bifunction $f$ is said to satisfy Lipschitz-type condition on $C$ if there exist two positive constants $c_{1}$ and $c_{2}$ such that

$$
f(x, y)+f(y, z) \geq f(x, z)-c_{1}\|x-y\|^{2}-c_{2}\|y-z\|^{2}, \forall x, y, z \in C .
$$

If $A: C \rightarrow H$ is a $L$-Lipschitz continuous operator, the bifunction $f(x, y)=\langle A x, y-x\rangle$ satisfies the Lipschitz-type condition with $c_{1}=c_{2}=L / 2$. Dealing with the analysis of the convergence of the proposed algorithms, we consider the following conditions imposed on a bifunction $f$ : $H \times H \rightarrow \mathfrak{R}:$

(A1) $f$ is pseudomonotone on $C$ and $f(x, x)=0$ for all $x \in C$;

(A2) $f$ satisfies Lipschitz-type condition on $H$ with some constants $c_{1}, c_{2}$;

(A3) $f(x,$.$) is convex and lower semicontinuos on C$ for every fixed $x \in H$;

(A4) $\limsup _{n \rightarrow \infty} f\left(x_{n}, y\right) \leq f(x, y)$ for each sequence $\left\{x_{n}\right\} \subset C$ converging weakly to $x$;

(A5) $E P(f, C)$ is nonempty;

It is easy to show that under assumptions (A1) and (A3), the solution set $E P(f, C)$ is closed and convex. Recall that the proximal mapping of a proper, convex and lower semicontinuous function $g: C \rightarrow \mathfrak{R}$ with a parameter $\lambda>0$ is defined by

$$
\operatorname{prox}_{\lambda g}(x)=\arg \min \left\{\lambda g(y)+\frac{1}{2}\|x-y\|^{2}: y \in C\right\}, x \in H .
$$

The following is a property of the proximal mapping. 
Lemma 2.1. [39] For each $x \in H$ and $\lambda>0$,

$$
\lambda\left\{g(y)-g\left(\operatorname{prox}_{\lambda g}(x)\right)\right\} \geq\left\langle x-\operatorname{prox}_{\lambda g}(x), y-\operatorname{prox}_{\lambda g}(x)\right\rangle, \forall y \in C .
$$

We need the following technical lemmas.

Lemma 2.2. [39] For all $x, y \in H$ and $\alpha \in \mathfrak{R}$, the following equality holds,

$$
\|\alpha x+(1-\alpha) y\|^{2}=\alpha\|x\|^{2}+(1-\alpha)\|y\|^{2}-\alpha(1-\alpha)\|x-y\|^{2} .
$$

Lemma 2.3. [21] Let $\left\{\Phi_{n}\right\},\left\{\Delta_{n}\right\}$ and $\left\{\theta_{n}\right\}$ be sequences in $[0,+\infty)$ such that

$$
\Phi_{n+1} \leq \Phi_{n}+\theta_{n}\left(\Phi_{n}-\Phi_{n-1}\right)+\Delta_{n}, \forall n \geq 1, \quad \sum_{n=1}^{+\infty} \Delta_{n}<+\infty,
$$

and there exists a real number $\theta$ with $0 \leq \theta_{n} \leq \theta<1$ for all $n \geq 0$. Then the following assertions hold:

(i) $\sum_{n=1}^{+\infty}\left[\Phi_{n}-\Phi_{n-1}\right]_{+}<+\infty$, where $[t]_{+}:=\max \{t, 0\}$;

(ii) There exists $\Phi^{*} \in[0,+\infty)$ such that $\lim _{n \rightarrow+\infty} \Phi_{n}=\Phi^{*}$.

Lemma 2.4. [39, Lemma 2.39] Let $C$ be a nonempty set of $H$ and $\left\{x_{n}\right\}$ be a sequence in $H$ such that the following two conditions hold:

(i) for every $x \in C, \lim _{n \rightarrow \infty}\left\|x_{n}-x\right\|$ exists;

(ii) every sequentially weak cluster point of $\left\{x_{n}\right\}$ is in $C$.

Then $\left\{x_{n}\right\}$ converges weakly to a point in $C$.

\section{Relaxed Inertial Extragradient Method}

In this section, we present a new relaxed inertial algorithm for solving problem (EP) in a Hilbert space. The algorithm can be considered as a combination between a relaxed version of the extragradient method and the inertial technique. Precisely, the algorithm is described as follows:

\section{Algorithm 1. [Relaxed Inertial Extragradient Method for EPs]}

Initialization: Choose $x_{0}, x_{1} \in H$ and three control parameter sequences $\left\{\lambda_{n}\right\} \subset(0,+\infty)$, $\left\{\alpha_{n}\right\} \subset(0,+\infty)$ and $\left\{\theta_{n}\right\} \subset[0,+\infty)$.

Iterative steps: Assume that $x_{n-1}, x_{n} \in H$ are known. Calculate $x_{n+1}$ as follows:

Step 1. Compute $w_{n}=x_{n}+\theta_{n}\left(x_{n}-x_{n-1}\right)$ and

$$
y_{n}=\operatorname{prox}_{\lambda_{n} f\left(w_{n}, .\right)}\left(w_{n}\right) .
$$

Step 2. Compute

$$
x_{n+1}=\left(1-\alpha_{n}\right) w_{n}+\alpha_{n} \operatorname{prox}_{\lambda_{n} f\left(y_{n}, .\right)}\left(w_{n}\right) .
$$

Set $n:=n+1$ and go back Step 1 .

Stopping criterion: If $y_{n}=w_{n}$ then stop and $y_{n}$ is a solution of problem (EP). 
Remark 3.1. In the case where problem (EP) is a variational inequality problem, i.e., $f(x, y)=$ $\langle A x, y-x\rangle$, where $A: H \rightarrow H$ is an operator, then Algorithm 1 becomes the following algorithm

$$
\left\{\begin{array}{l}
w_{n}=x_{n}+\theta_{n}\left(x_{n}-x_{n-1}\right), \\
y_{n}=P_{C}\left(w_{n}-\lambda_{n} A w_{n}\right), \\
x_{n+1}=\left(1-\alpha_{n}\right) w_{n}+\alpha_{n} P_{C}\left(w_{n}-\lambda_{n} A y_{n}\right) .
\end{array}\right.
$$

If $\theta_{n}=0$, then $w_{n}=x_{n}$ and (3.1) reduces to the algorithm

$$
\left\{\begin{array}{l}
y_{n}=P_{C}\left(x_{n}-\lambda_{n} A x_{n}\right), \\
x_{n+1}=\left(1-\alpha_{n}\right) x_{n}+\alpha_{n} P_{C}\left(x_{n}-\lambda_{n} A y_{n}\right),
\end{array}\right.
$$

which is a relaxed version of the extragradient method in [37].

In order to establish the convergence of Algorithm 1, we assume that conditions (A1)-(A5) hold, and that three parameter sequences $\left\{\lambda_{n}\right\},\left\{\alpha_{n}\right\},\left\{\theta_{n}\right\}$ satisfy the following conditions:

(B1) $0<\lambda_{*} \leq \lambda_{n} \leq \lambda^{*}<\min \left\{\frac{1}{2 c_{1}}, \frac{1}{2 c_{2}}\right\}$;

(B2) $0<\alpha_{*} \leq \alpha_{n} \leq \alpha^{*} \leq \frac{1}{2}+\frac{1}{4} \min \left\{1-2 c_{1} \lambda^{*}, 1-2 c_{2} \lambda^{*}\right\}$;

(B3) $0 \leq \theta_{n} \leq \theta<\frac{\varepsilon}{2 \varepsilon+1}$ and $\left\{\theta_{n}\right\}$ is non-decreasing, where

$$
\varepsilon:=\frac{0.5 \min \left\{1-2 c_{1} \lambda^{*}, 1-2 c_{2} \lambda^{*}\right\}+1-\alpha^{*}}{\alpha^{*}} .
$$

Remark 3.2. From (B2), we have $2 \alpha^{*} \leq 1+0.5 \min \left\{1-2 c_{1} \lambda^{*}, 1-2 c_{2} \lambda^{*}\right\}$. Thus

$$
0.5 \min \left\{1-2 c_{1} \lambda^{*}, 1-2 c_{2} \lambda^{*}\right\}+1-\alpha^{*} \geq \alpha^{*} .
$$

This implies $\varepsilon \geq 1$.

We begin with the following lemma.

Lemma 3.1. Under assumptions (A1)-(A3), (A5) and (B1)-(B3), the following estimate holds for all $n \geq 0$ and $x^{*} \in E P(f, C)$,

$$
\left\|x_{n+1}-x^{*}\right\|^{2} \leq\left\|w_{n}-x^{*}\right\|^{2}-\frac{\kappa_{n}+1-\alpha_{n}}{\alpha_{n}}\left\|x_{n+1}-w_{n}\right\|^{2},
$$

where $\kappa_{n}=0.5 \min \left\{1-2 \lambda_{n} c_{1}, 1-2 \lambda_{n} c_{2}\right\}$.

Proof. Set $z_{n}=\operatorname{prox}_{\lambda_{n} f\left(y_{n}, .\right)}\left(w_{n}\right)$. Thus, from Lemma 2.1, we obtain

$$
\lambda_{n}\left(f\left(y_{n}, y\right)-f\left(y_{n}, z_{n}\right)\right) \geq\left\langle w_{n}-z_{n}, y-z_{n}\right\rangle, \forall y \in C .
$$

Similarly, by the definition of $y_{n}$ and Lemma 2.1, we get

$$
\lambda_{n}\left(f\left(w_{n}, y\right)-f\left(w_{n}, y_{n}\right)\right) \geq\left\langle w_{n}-y_{n}, y-y_{n}\right\rangle, \forall y \in C .
$$

Substituting $y=z_{n}$ into relation (3.3), we obtain

$$
\lambda_{n}\left(f\left(w_{n}, z_{n}\right)-f\left(w_{n}, y_{n}\right)\right) \geq\left\langle w_{n}-y_{n}, z_{n}-y_{n}\right\rangle .
$$

Now, since $x^{*} \in E P(f, C)$, we have $f\left(x^{*}, y_{n}\right) \geq 0$. Thus, from hypothesis (A1), we obtain $f\left(y_{n}, x^{*}\right) \leq 0$. Next, substituting $y=x^{*} \in E P(f, C) \subset C$ into relation (3.2) and using $f\left(y_{n}, x^{*}\right) \leq$ 0 , we see that

$$
-\lambda_{n} f\left(y_{n}, z_{n}\right) \geq\left\langle w_{n}-z_{n}, x^{*}-z_{n}\right\rangle
$$


Thus $\left\langle w_{n}-z_{n}, z_{n}-x^{*}\right\rangle \geq \lambda_{n} f\left(y_{n}, z_{n}\right)$, which together with the Lipschitz-type condition of $f$, implies that

$$
\left\langle w_{n}-z_{n}, z_{n}-x^{*}\right\rangle \geq \lambda_{n}\left\{f\left(w_{n}, z_{n}\right)-f\left(w_{n}, y_{n}\right)-c_{1}\left\|w_{n}-y_{n}\right\|^{2}-c_{2}\left\|y_{n}-z_{n}\right\|^{2}\right\} .
$$

Combining the last inequality with relation (3.4), we obtain

$$
\left\langle w_{n}-z_{n}, z_{n}-x^{*}\right\rangle \geq\left\langle w_{n}-y_{n}, z_{n}-y_{n}\right\rangle-c_{1} \lambda_{n}\left\|w_{n}-y_{n}\right\|^{2}-c_{2} \lambda_{n}\left\|y_{n}-z_{n}\right\|^{2} .
$$

We have the following facts:

$$
\begin{aligned}
& 2\left\langle w_{n}-z_{n}, z_{n}-x^{*}\right\rangle=\left\|w_{n}-x^{*}\right\|^{2}-\left\|w_{n}-z_{n}\right\|^{2}-\left\|z_{n}-x^{*}\right\|^{2}, \\
& 2\left\langle w_{n}-y_{n}, z_{n}-y_{n}\right\rangle=\left\|w_{n}-y_{n}\right\|^{2}+\left\|z_{n}-y_{n}\right\|^{2}-\left\|w_{n}-z_{n}\right\|^{2} .
\end{aligned}
$$

Multiplying both two sides of inequality (3.5) by 2 , after that, using the two last equalities, we obtain

$$
\left\|z_{n}-x^{*}\right\|^{2} \leq\left\|w_{n}-x^{*}\right\|^{2}-\left(1-2 \lambda_{n} c_{1}\right)\left\|y_{n}-w_{n}\right\|^{2}-\left(1-2 \lambda_{n} c_{2}\right)\left\|z_{n}-y_{n}\right\|^{2} .
$$

Thus, from the definition of $\kappa_{n}$, we get

$$
\begin{aligned}
\left\|z_{n}-x^{*}\right\|^{2} & \leq\left\|w_{n}-x^{*}\right\|^{2}-2 \kappa_{n}\left(\left\|y_{n}-w_{n}\right\|^{2}+\left\|z_{n}-y_{n}\right\|^{2}\right) \\
& \leq\left\|w_{n}-x^{*}\right\|^{2}-\kappa_{n}\left(\left\|y_{n}-w_{n}\right\|+\left\|z_{n}-y_{n}\right\|\right)^{2} \\
& \leq\left\|w_{n}-x^{*}\right\|^{2}-\kappa_{n}\left\|z_{n}-w_{n}\right\|^{2} .
\end{aligned}
$$

Note that, from the definitions of $x_{n+1}$ and $z_{n}$, one has $x_{n+1}=\left(1-\alpha_{n}\right) w_{n}+\alpha_{n} z_{n}$. Moreover, two hypotheses (B1) and (B2) imply that $\alpha_{n} \in(0,1)$. Thus, it follows from Lemma 2.2 and relation $(3.7)$ that

$$
\begin{aligned}
\left\|x_{n+1}-x^{*}\right\|^{2} & =\left\|\left(1-\alpha_{n}\right)\left(w_{n}-x^{*}\right)+\alpha_{n}\left(z_{n}-x^{*}\right)\right\|^{2} \\
& =\left(1-\alpha_{n}\right)\left\|w_{n}-x^{*}\right\|^{2}+\alpha_{n}\left\|z_{n}-x^{*}\right\|^{2}-\alpha_{n}\left(1-\alpha_{n}\right)\left\|z_{n}-w_{n}\right\|^{2} \\
& \leq\left\|w_{n}-x^{*}\right\|^{2}-\alpha_{n}\left(\kappa_{n}+1-\alpha_{n}\right)\left\|z_{n}-w_{n}\right\|^{2},
\end{aligned}
$$

which, together with the fact $\left\|z_{n}-w_{n}\right\|=\frac{1}{\alpha_{n}}\left\|x_{n+1}-w_{n}\right\|$, implies that

$$
\left\|x_{n+1}-x^{*}\right\|^{2} \leq\left\|w_{n}-x^{*}\right\|^{2}-\frac{\kappa_{n}+1-\alpha_{n}}{\alpha_{n}}\left\|x_{n+1}-w_{n}\right\|^{2} \text {. }
$$

This completes the proof of Lemma 3.1.

Now, we set

$$
\varphi_{n}:=\left\|x_{n}-x^{*}\right\|^{2}-\theta_{n}|| x_{n-1}-x^{*}\left\|^{2}+\theta_{n}(1+\varepsilon)\right\| x_{n}-x_{n-1} \|^{2},
$$

where $\varepsilon$ is defined in hypothesis (B3). Let $K:=\varepsilon-(2 \varepsilon+1) \theta$. From (B3), we see that $K>0$. We have the following lemma.

Lemma 3.2. Under assumptions as in Lemma 3.1, the following estimate holds, for all $n \geq 1$,

$$
\varphi_{n+1}-\varphi_{n} \leq-K\left\|x_{n+1}-x_{n}\right\|^{2} .
$$

Proof. It follows from (B1)-(B2) and the definition of $\kappa_{n}$ that

$$
\begin{aligned}
\frac{\kappa_{n}+1-\alpha_{n}}{\alpha_{n}} & =\frac{0.5 \min \left\{1-2 \lambda_{n} c_{1}, 1-2 \lambda_{n} c_{2}\right\}+1-\alpha_{n}}{\alpha_{n}} \\
& \geq \frac{0.5 \min \left\{1-2 \lambda^{*} c_{1}, 1-2 \lambda^{*} c_{2}\right\}+1-\alpha^{*}}{\alpha^{*}}=\varepsilon .
\end{aligned}
$$


Thus, from Lemma 3.1, we obtain

$$
\left\|x_{n+1}-x^{*}\right\|^{2} \leq\left\|w_{n}-x^{*}\right\|^{2}-\varepsilon\left\|x_{n+1}-w_{n}\right\|^{2} .
$$

By the definition of $w_{n}$ and Lemma 2.2, we have

$$
\begin{aligned}
\left\|w_{n}-x^{*}\right\|^{2} & =\left\|x_{n}+\theta_{n}\left(x_{n}-x_{n-1}\right)-x^{*}\right\|^{2} \\
& =\left\|\left(1+\theta_{n}\right)\left(x_{n}-x^{*}\right)+\left(-\theta_{n}\right)\left(x_{n-1}-x^{*}\right)\right\|^{2} \\
& =\left(1+\theta_{n}\right)\left\|x_{n}-x^{*}\right\|^{2}-\theta_{n}\left\|x_{n-1}-x^{*}\right\|^{2}+\theta_{n}\left(1+\theta_{n}\right)\left\|x_{n}-x_{n-1}\right\|^{2} .
\end{aligned}
$$

Moreover, it also follows from the definition of $w_{n}$ and the Cauchy-Schwarz inequality that

$$
\begin{aligned}
\left\|x_{n+1}-w_{n}\right\|^{2} & =\left\|x_{n+1}-x_{n}-\theta_{n}\left(x_{n}-x_{n-1}\right)\right\|^{2} \\
& =\left\|x_{n+1}-x_{n}\right\|^{2}+\theta_{n}^{2}\left\|x_{n}-x_{n-1}\right\|^{2}-2 \theta_{n}\left\langle x_{n+1}-x_{n}, x_{n}-x_{n-1}\right\rangle \\
& \geq\left\|x_{n+1}-x_{n}\right\|^{2}+\theta_{n}^{2}\left\|x_{n}-x_{n-1}\right\|^{2}-2 \theta_{n}\left\|x_{n+1}-x_{n}\right\|\left\|\mid x_{n}-x_{n-1}\right\| \\
& \geq\left\|x_{n+1}-x_{n}\right\|^{2}+\theta_{n}^{2}\left\|x_{n}-x_{n-1}\right\|^{2}-\theta_{n}\left[\left\|x_{n+1}-x_{n}\right\|^{2}+\left\|x_{n}-x_{n-1}\right\|^{2}\right] \\
& =\left(1-\theta_{n}\right)\left\|x_{n+1}-x_{n}\right\|^{2}+\left(\theta_{n}^{2}-\theta_{n}\right)\left\|x_{n}-x_{n-1}\right\|^{2} .
\end{aligned}
$$

From relations (3.9) - (3.11), we have

$$
\begin{aligned}
\left\|x_{n+1}-x^{*}\right\|^{2} \leq & \left(1+\theta_{n}\right)\left\|x_{n}-x^{*}\right\|^{2}-\theta_{n}\left\|x_{n-1}-x^{*}\right\|^{2}-\varepsilon\left(1-\theta_{n}\right)\left\|x_{n+1}-x_{n}\right\|^{2} \\
& -\varepsilon\left(\theta_{n}^{2}-\theta_{n}\right)\left\|x_{n}-x_{n-1}\right\|^{2}+\theta_{n}\left(1+\theta_{n}\right)\left\|x_{n}-x_{n-1}\right\|^{2} \\
\leq & \left(1+\theta_{n}\right)\left\|x_{n}-x^{*}\right\|^{2}-\theta_{n}\left\|x_{n-1}-x^{*}\right\|^{2}-\varepsilon\left(1-\theta_{n}\right)\left\|x_{n+1}-x_{n}\right\|^{2} \\
& +\theta_{n}\left(1+\varepsilon-\theta_{n}(\varepsilon-1)\right)\left\|x_{n}-x_{n-1}\right\|^{2} \\
\leq & \left(1+\theta_{n}\right)\left\|x_{n}-x^{*}\right\|^{2}-\theta_{n}\left\|x_{n-1}-x^{*}\right\|^{2}-\varepsilon\left(1-\theta_{n}\right)\left\|x_{n+1}-x_{n}\right\|^{2} \\
& +\theta_{n}(1+\varepsilon)\left\|x_{n}-x_{n-1}\right\|^{2},
\end{aligned}
$$

in which the last inequality follows from the fact that $\varepsilon \geq 1$ and $\theta_{n} \geq 0$. Now, it follows from the definition of $\varphi_{n}$, the non-decreasing property of $\left\{\theta_{n}\right\}$, and relation (3.12) that

$$
\begin{aligned}
\varphi_{n+1}-\varphi_{n}= & \left\|x_{n+1}-x^{*}\right\|^{2}-\left(1+\theta_{n+1}\right)\left\|x_{n}-x^{*}\right\|^{2}+\theta_{n+1}(1+\varepsilon)\left\|x_{n+1}-x_{n}\right\|^{2} \\
& +\theta_{n}\left\|x_{n-1}-x^{*}\right\|^{2}-\theta_{n}(1+\varepsilon)\left\|x_{n}-x_{n-1}\right\|^{2} \\
\leq & \left\|x_{n+1}-x^{*}\right\|^{2}-\left(1+\theta_{n}\right)\left\|x_{n}-x^{*}\right\|^{2}+\theta_{n}\left\|x_{n-1}-x^{*}\right\|^{2} \\
& -\theta_{n}(1+\varepsilon)\left\|x_{n}-x_{n-1}\right\|^{2}+\theta_{n+1}(1+\varepsilon)\left\|x_{n+1}-x_{n}\right\|^{2} \\
\leq & -\varepsilon\left(1-\theta_{n}\right)\left\|x_{n+1}-x_{n}\right\|^{2}+\theta_{n+1}(1+\varepsilon)\left\|x_{n+1}-x_{n}\right\|^{2} \\
= & -\left[\varepsilon\left(1-\theta_{n}\right)-\theta_{n+1}(1+\varepsilon)\right]\left\|x_{n+1}-x_{n}\right\|^{2} \\
\leq & -[\varepsilon(1-\theta)-\theta(1+\varepsilon)]\left\|x_{n+1}-x_{n}\right\|^{2}\left(\text { due to } 0 \leq \theta_{n}, \theta_{n+1} \leq \theta\right) \\
= & -[\varepsilon-(2 \varepsilon+1) \theta]\left\|x_{n+1}-x_{n}\right\|^{2}=-K\left\|x_{n+1}-x_{n}\right\|^{2} .
\end{aligned}
$$

Lemma 3.2 is proved.

Lemma 3.3. Under assumptions as in Lemma 3.1, the following assertions hold:

(i) $\sum_{n=1}^{\infty}\left\|x_{n+1}-x_{n}\right\|^{2}<+\infty$;

(ii) $\lim _{n \rightarrow \infty}|| x_{n}-y_{n}||=\lim _{n \rightarrow \infty}|| z_{n}-w_{n}\left\|=\lim _{n \rightarrow \infty}|| y_{n}-w_{n}\right\|=\lim _{n \rightarrow \infty}|| y_{n}-z_{n} \|=0$;

(iii) $\lim _{n \rightarrow \infty}\left\|x_{n}-x^{*}\right\|^{2} \in \mathfrak{R}$ for each $x^{*} \in E P(f, C)$ and $\left\{x_{n}\right\},\left\{y_{n}\right\},\left\{z_{n}\right\},\left\{w_{n}\right\}$ are bounded. 
Proof. (i) It follows from Lemma 3.2 that $\varphi_{n}$ is a non-increasing. Thus, from the definition of $\varphi_{n}$, we have

$$
\left\|x_{n}-x^{*}\right\|^{2} \leq \theta_{n}\left\|x_{n-1}-x^{*}\right\|^{2}+\varphi_{n} \leq \theta_{n}\left\|x_{n-1}-x^{*}\right\|^{2}+\varphi_{1} .
$$

Thus, from hypothesis (B3), we get

$$
\left\|x_{n}-x^{*}\right\|^{2} \leq \theta\left\|x_{n-1}-x^{*}\right\|^{2}+\varphi_{1}
$$

This implies that

$$
\left\|x_{n}-x^{*}\right\|^{2} \leq \theta^{n}|| x_{0}-x^{*}\left\|^{2}+\varphi_{1}\left(\theta^{n-1}+\theta^{n-2}+\ldots+\theta+1\right) \leq \theta^{n}\right\| x_{0}-x^{*} \|^{2}+\frac{\varphi_{1}}{1-\theta} .
$$

Moreover,

$$
\varphi_{n+1}=\left\|x_{n+1}-x^{*}\right\|^{2}-\theta_{n+1}\left\|x_{n}-x^{*}\right\|^{2}+\theta_{n+1}(1+\varepsilon)\left\|x_{n+1}-x_{n}\right\|^{2} \geq-\theta_{n+1}\left\|x_{n}-x^{*}\right\|^{2} .
$$

This together with (3.13) implies that

$$
-\varphi_{n+1} \leq \theta_{n+1}\left\|x_{n}-x^{*}\right\|^{2} \leq \theta\left\|x_{n}-x^{*}\right\|^{2} \leq \theta^{n+1}\left\|x_{0}-x^{*}\right\|^{2}+\frac{\theta \varphi_{1}}{1-\theta} .
$$

It follows from Lemma 3.2 that

$$
K\left\|x_{n+1}-x_{n}\right\|^{2} \leq \varphi_{n}-\varphi_{n+1}
$$

for all $n \geq 1$. Let a fixed $N \geq 1$. Applying the last inequality for $n=1,2, \ldots, N$ and summing up those inequalities, we obtain

$$
K \sum_{n=1}^{N}\left\|x_{n+1}-x_{n}\right\|^{2} \leq \varphi_{1}-\varphi_{N+1}
$$

Combining this with relation (3.14), we obtain

$$
K \sum_{n=1}^{N}\left\|x_{n+1}-x_{n}\right\|^{2} \leq \varphi_{1}+\theta^{N+1}\left\|x_{0}-x^{*}\right\|^{2}+\frac{\theta \varphi_{1}}{1-\theta} \leq \theta^{N+1}\left\|x_{0}-x^{*}\right\|^{2}+\frac{\varphi_{1}}{1-\theta} .
$$

From (B3), we obtain $\theta \leq \frac{\varepsilon}{2 \varepsilon+1}<1$. Thus, passing to the limit in the last inequality as $N \rightarrow \infty$ and noting that $K>0$, we get

$$
\sum_{n=1}^{\infty}\left\|x_{n+1}-x_{n}\right\|^{2} \leq \frac{\varphi_{1}}{1-\theta}<\infty .
$$

(ii) It follows from relation (3.15) that $\left\|x_{n+1}-x_{n}\right\| \rightarrow 0$. Hence

$$
\left\|x_{n+1}-w_{n}\right\|=\left\|x_{n+1}-x_{n}+\theta_{n}|| x_{n}-x_{n-1}\right\| \leq\left\|x_{n+1}-x_{n}\right\|+\theta_{n}\left\|x_{n}-x_{n-1}\right\| \rightarrow 0 .
$$

It is obvious that $\left\|x_{n}-w_{n}\right\| \leq\left\|x_{n}-x_{n+1}\right\|+\left\|x_{n+1}-w_{n}\right\| \rightarrow 0$. Moreover, from the defition of $x_{n+1}$, we also have

$$
\left\|z_{n}-w_{n}\right\|=\frac{1}{\alpha_{n}}\left\|x_{n+1}-w_{n}\right\| \rightarrow 0
$$

because $\alpha_{n} \geq \alpha_{*}>0$. It follows from (3.12) that

$$
\left\|x_{n+1}-x^{*}\right\|^{2} \leq\left(1+\theta_{n}\right)\left\|x_{n}-x^{*}\right\|^{2}-\theta_{n}\left\|x_{n-1}-x^{*}\right\|^{2}+\theta_{n}(1+\varepsilon)\left\|x_{n}-x_{n-1}\right\|^{2} .
$$

Thus, by setting $\Phi_{n}=\left\|x_{n}-x^{*}\right\|^{2}$ and $\Delta_{n}=\theta_{n}(1+\varepsilon)|| x_{n}-x_{n-1} \|^{2}$, we obtain

$$
\Phi_{n+1} \leq \Phi_{n}+\theta_{n}\left(\Phi_{n}-\Phi_{n-1}\right)+\Delta_{n} \forall n \geq 1 \text {. }
$$


It follows from $0 \leq \theta_{n} \leq \theta \leq \frac{\varepsilon}{2 \varepsilon+1}<1$, the definition of $\Delta_{n}$ and relation (3.15) that $\sum_{n=1}^{+\infty} \Delta_{n}<$ $+\infty$. Thus, Lemma 2.3 and relation (3.16) ensure that the limit of $\left\{\Phi_{n}\right\}$ exists, i.e., $\lim _{n \rightarrow \infty} \| x_{n}-$ $x^{*} \|^{2}=\Phi^{*} \in \Re$. We also have $\lim _{n \rightarrow \infty}\left\|w_{n}-x^{*}\right\|^{2}=\lim _{n \rightarrow \infty}\left\|z_{n}-x^{*}\right\|^{2}=\Phi^{*}$ because of $\| x_{n}-$ $w_{n} \| \rightarrow 0$ and $\left\|z_{n}-w_{n}\right\| \rightarrow 0$. Thus, it follows from relation (3.6) that

$$
\left(1-2 \lambda_{n} c_{1}\right)\left\|y_{n}-w_{n}\right\|^{2}+\left(1-2 \lambda_{n} c_{2}\right)\left\|z_{n}-y_{n}\right\|^{2} \leq\left\|w_{n}-x^{*}\right\|^{2}-\left\|z_{n}-x^{*}\right\|^{2} \rightarrow 0 .
$$

This together with hypothesis (B1) implies that

$$
\left\|y_{n}-w_{n}\right\| \rightarrow 0, \quad\left\|z_{n}-y_{n}\right\| \rightarrow 0 .
$$

Thus, we also have $\left\|x_{n}-y_{n}\right\| \rightarrow 0$ due to $\left\|x_{n}-w_{n}\right\| \rightarrow 0$.

(iii) Since $\lim _{n \rightarrow \infty}\left\|x_{n}-x^{*}\right\|^{2}=\Phi^{*}$, we easily see that $\left\{x_{n}\right\}$ is bounded. Therefore $\left\{y_{n}\right\},\left\{z_{n}\right\}$ and $\left\{w_{n}\right\}$ are also bounded.

Lemma 3.4. Under assumptions (A1)-(A5) and (B1)-(B3), every weakly cluster point of $\left\{x_{n}\right\}$ belongs to $\operatorname{EP}(f, C)$.

Proof. From relation (3.2), we have

$$
\left.\lambda_{n} f\left(y_{n}, y\right) \geq \lambda_{n} f\left(y_{n}, z_{n}\right)\right)+\left\langle w_{n}-z_{n}, y-z_{n}\right\rangle, \forall y \in C .
$$

It follows from the Lipschitz-type condition of $f$ that

$$
f\left(y_{n}, z_{n}\right) \geq f\left(w_{n}, z_{n}\right)-f\left(w_{n}, y_{n}\right)-c_{1}\left\|w_{n}-y_{n}\right\|^{2}-c_{2}\left\|y_{n}-z_{n}\right\|^{2} .
$$

Multiplying both two sides of this inequality by $\lambda_{n}>0$, after that, combining the obtained inequality with relation (3.4), we get

$$
\lambda_{n} f\left(y_{n}, z_{n}\right) \geq\left\langle w_{n}-y_{n}, z_{n}-y_{n}\right\rangle-c_{1} \lambda_{n}\left\|w_{n}-y_{n}\right\|^{2}-c_{2} \lambda_{n}\left\|y_{n}-z_{n}\right\|^{2},
$$

which, together with relation (3.19), implies that

$$
\begin{aligned}
\lambda_{n} f\left(y_{n}, y\right) \geq & \left\langle w_{n}-y_{n}, z_{n}-y_{n}\right\rangle+\left\langle w_{n}-z_{n}, y-z_{n}\right\rangle \\
& -c_{1} \lambda_{n}\left\|w_{n}-y_{n}\right\|^{2}-c_{2} \lambda_{n}|| y_{n}-z_{n} \|^{2}
\end{aligned}
$$

for all $y \in C$ and $n \geq 0$. Thus, passing to the limit in (3.20) as $n \rightarrow \infty$, and using Lemma 3.3(ii-iii) and (B1), we obtain

$$
\lim _{n \rightarrow \infty} f\left(y_{n}, y\right) \geq 0, \forall y \in C .
$$

Note that, from Lemma 3.3(iii), $\left\{x_{n}\right\}$ is bounded. Now, assume that $p$ is some weakly cluster point of $\left\{x_{n}\right\}$, i.e., there exists a subsequence $\left\{x_{m}\right\}$ of $\left\{x_{n}\right\}$ converging weakly to $p$. From $\left\|x_{m}-y_{m}\right\| \rightarrow 0$, we also obtain $y_{m} \rightarrow p$. Since $C$ is closed and convex in $H$, we have that $C$ is weakly closed. Thus, from $\left\{y_{m}\right\} \subset C$, we obtain $p \in C$. It follows from (A5) and relation (3.21) that

$$
f(p, y) \geq \lim _{m \rightarrow \infty} \sup f\left(y_{m}, y\right) \geq 0, \forall y \in C .
$$

This means that $p \in E P(f, C)$. The proof of Lemma 3.4 is complete.

Finally, we obtain the following main result.

Theorem 3.1. Under assumptions (A1)-(A5) and (B1)-(B3), the sequences $\left\{x_{n}\right\},\left\{y_{n}\right\}$ and $\left\{w_{n}\right\}$ generated by Algorithm 1 converge weakly to some solution of problem (EP). 
Proof. From Lemmas 2.4, 3.3 (iii) and 3.4, we obtain that $\left\{x_{n}\right\}$ converges weakly to some solution $x^{\dagger}$ of problem (EP). From Lemma 3.3 (ii), we have that $\left\{y_{n}\right\}$ and $\left\{w_{n}\right\}$ also converge weakly to $x^{\dagger}$. This completes the proof of Theorem 3.1.

\section{Modified Relaxed Inertial Extragradient Method}

In view of hypothesis (B1), we see that the stepsize $\lambda_{n}$ depends on the two Lipschitz-type constants $c_{1}, c_{2}$ of bifunction $f$. This means that the convergence of Algorithm 1 is only ensured when these constants are known. Actually, the Lipschitz-type constants are often unknown or difficult to approximate in nonlinear problems. In that case, a method of linesearch type can be used to replace. A linesearch involves an inner loop with some finite stopping criterion and requires many extra-computations at each (outer) iteration. This can be expensive and time-consuming. In this section, we introduce a modified version of Algorithm 1 where can be implemented more easily. For the presentation of Algorithm 2 below, we use the notation $[t]_{+}=\max \{0, t\}$ and adopt the convention $\frac{a}{0}=+\infty$ for $a \geq 0$.

\section{Algorithm 2. [Modified Relaxed Inertial Extragradient Method for EPs]}

Initialization: Choose $x_{0}, x_{1} \in H$. Take two constants $\lambda_{0}>0, \mu \in(0,1)$ and two control parameter sequences, $\left\{\alpha_{n}\right\} \subset(0,+\infty),\left\{\theta_{n}\right\} \subset[0,+\infty)$.

Iterative steps: Assume that $x_{n-1}, x_{n} \in H$ and $\lambda_{n}$ are known. Calculate $x_{n+1}$ and $\lambda_{n+1}$ as follows:

Step 1. Compute $w_{n}=x_{n}+\theta_{n}\left(x_{n}-x_{n-1}\right)$ and

$$
y_{n}=\operatorname{prox}_{\lambda_{n} f\left(w_{n}, .\right)}\left(w_{n}\right) .
$$

Step 2. Compute

$$
x_{n+1}=\left(1-\alpha_{n}\right) w_{n}+\alpha_{n} \operatorname{prox}_{\lambda_{n} f\left(y_{n}, .\right)}\left(w_{n}\right) .
$$

Step 3. Update

$$
\lambda_{n+1}=\min \left\{\lambda_{n}, \frac{\mu\left(\left\|w_{n}-y_{n}\right\|^{2}+\left\|z_{n}-y_{n}\right\|^{2}\right)}{2\left[f\left(w_{n}, z_{n}\right)-f\left(w_{n}, y_{n}\right)-f\left(y_{n}, z_{n}\right)\right]_{+}}\right\},
$$

where $z_{n}=\operatorname{prox}_{\lambda_{n} f\left(y_{n}, .\right)}\left(w_{n}\right)$. Set $n:=n+1$ and go back Step 1 .

Stopping criterion: If $y_{n}=w_{n}$ then stop and $y_{n}$ is a solution of problem (EP).

The main difference between Algorithm 2 and Algorithm 1 is at Step 3. The stepsize $\lambda_{n+1}$ is updated at each iteration based on the previous iterates, by a simple computation, and without a linesearch. Although the convergence of Algorithm 2 requires the Lipschitz-type condition of bifunction $f$, the Lipschitz-type constants of $f$ is not necessary to be known. In order to establish the convergence of Algorithm 2, we consider the following assumptions imposed on the two sequences $\left\{\alpha_{n}\right\},\left\{\theta_{n}\right\}$ :

(B4) $0<\alpha_{*} \leq \alpha_{n} \leq \alpha^{*}<\frac{3-\mu}{4}$; 
(B5) $0 \leq \theta_{n} \leq \theta<\frac{\bar{\varepsilon}}{2 \bar{\varepsilon}+1}$ and $\left\{\theta_{n}\right\}$ is non-decreasing, where $\bar{\varepsilon} \in\left[1, \frac{3-\mu-2 \alpha^{*}}{2 \alpha^{*}}\right)$.

Remark 4.1. Since $0<\alpha^{*}<\frac{3-\mu}{4}$, we immediately obtain $\frac{3-\mu-2 \alpha^{*}}{2 \alpha^{*}}>1$.

Theorem 4.1. Under assumptions (A1)-(A5) and (B4)-(B5), the sequences $\left\{x_{n}\right\},\left\{y_{n}\right\}$ and $\left\{w_{n}\right\}$ generated by Algorithm 2 converge weakly to some solution of problem (EP).

Proof. We first state that $\lambda_{n}>0$ for all $n \geq 0$ and

$$
f\left(w_{n}, z_{n}\right)-f\left(w_{n}, y_{n}\right)-f\left(y_{n}, z_{n}\right) \leq \frac{\mu\left(\left\|w_{n}-y_{n}\right\|^{2}+\left\|z_{n}-y_{n}\right\|^{2}\right)}{2 \lambda_{n+1}}, \forall n \geq 1 .
$$

Indeed, we see that $\lambda_{0}>0$. Assume $\lambda_{n}>0$ for some $n \geq 0$. Now, if $f\left(w_{n}, z_{n}\right)-f\left(w_{n}, y_{n}\right)-$ $f\left(y_{n}, z_{n}\right) \leq 0$, then from the definition of $\lambda_{n+1}$, we obtain that $\lambda_{n+1}=\lambda_{n}>0$, and inequality (4.1) is obviously true. In the inverse case, if $f\left(w_{n}, z_{n}\right)-f\left(w_{n}, y_{n}\right)-f\left(y_{n}, z_{n}\right)>0$, then $\| w_{n}-$ $y_{n}\left\|^{2}+\right\| z_{n}-y_{n} \|^{2}>0$ and

$$
\left[f\left(w_{n}, z_{n}\right)-f\left(w_{n}, y_{n}\right)-f\left(y_{n}, z_{n}\right)\right]_{+}=f\left(w_{n}, z_{n}\right)-f\left(w_{n}, y_{n}\right)-f\left(y_{n}, z_{n}\right)>0 .
$$

Thus, it follows from the definition of $\lambda_{n+1}$ that $\lambda_{n+1}>0$ and

$$
\lambda_{n+1} \leq \frac{\mu\left(\left\|w_{n}-y_{n}\right\|^{2}+\left\|z_{n}-y_{n}\right\|^{2}\right)}{2\left(f\left(w_{n}, z_{n}\right)-f\left(w_{n}, y_{n}\right)-f\left(y_{n}, z_{n}\right)\right)},
$$

which follows inequality (4.1). Moreover, using the result in [9, Theorem 1] and hypothesis (A2), we obtain

$$
\lim \lambda_{n}=\lambda>0
$$

Now, we obtain from Lemma 2.1 and the definition of $z_{n}$ that

$$
\lambda_{n}\left(f\left(y_{n}, x^{*}\right)-f\left(y_{n}, z_{n}\right)\right) \geq\left\langle w_{n}-z_{n}, x^{*}-z_{n}\right\rangle, \forall x^{*} \in E P(f, C) .
$$

Similarly, it follows from the definition of $y_{n}$ that

$$
\lambda_{n}\left(f\left(w_{n}, z_{n}\right)-f\left(w_{n}, y_{n}\right)\right) \geq\left\langle w_{n}-y_{n}, z_{n}-y_{n}\right\rangle .
$$

Combining relation (4.3) and (4.4) and noting $f\left(y_{n}, x^{*}\right) \leq 0$, we derive

$$
2\left\langle w_{n}-z_{n}, x^{*}-z_{n}\right\rangle+2\left\langle w_{n}-y_{n}, z_{n}-y_{n}\right\rangle \leq 2 \lambda_{n}\left(f\left(w_{n}, z_{n}\right)-f\left(w_{n}, y_{n}\right)-f\left(y_{n}, z_{n}\right)\right),
$$

which together with relation (4.1) implies that

$$
2\left\langle w_{n}-z_{n}, x^{*}-z_{n}\right\rangle+2\left\langle w_{n}-y_{n}, z_{n}-y_{n}\right\rangle \leq \frac{\mu \lambda_{n}}{\lambda_{n+1}}\left(\left\|w_{n}-y_{n}\right\|^{2}+\left\|z_{n}-y_{n}\right\|^{2}\right) .
$$

Applying the equality $2\langle a, b\rangle=\|a\|^{2}+\|b\|^{2}-\|a-b\|^{2}$ to relation (4.5), we come to the following inequality

$$
\left\|z_{n}-x^{*}\right\|^{2} \leq\left\|w_{n}-x^{*}\right\|^{2}-\left(1-\frac{\mu \lambda_{n}}{\lambda_{n+1}}\right)\left(\left\|w_{n}-y_{n}\right\|^{2}+\left\|z_{n}-y_{n}\right\|^{2}\right) .
$$

Thus, from the fact $\left(a^{2}+b^{2}\right) \geq \frac{1}{2}(a+b)^{2}$ and the triangle inequality, we get

$$
\begin{aligned}
\left\|z_{n}-x^{*}\right\|^{2} & \leq\left\|w_{n}-x^{*}\right\|^{2}-\frac{1}{2}\left(1-\frac{\mu \lambda_{n}}{\lambda_{n+1}}\right)\left(\left\|w_{n}-y_{n}\right\|+\left\|z_{n}-y_{n}\right\|\right)^{2} \\
& \leq\left\|w_{n}-x^{*}\right\|^{2}-\frac{1}{2}\left(1-\frac{\mu \lambda_{n}}{\lambda_{n+1}}\right)\left\|z_{n}-w_{n}\right\|^{2} \\
& =\left\|w_{n}-x^{*}\right\|^{2}-\bar{\kappa}_{n}\left\|z_{n}-w_{n}\right\|^{2},
\end{aligned}
$$


where $\bar{\kappa}_{n}=\frac{1}{2}\left(1-\frac{\mu \lambda_{n}}{\lambda_{n+1}}\right)$. Thus, as relation (3.8), we also obtain

$$
\left\|x_{n+1}-x^{*}\right\|^{2} \leq\left\|w_{n}-x^{*}\right\|^{2}-\frac{\bar{\kappa}_{n}+1-\alpha_{n}}{\alpha_{n}}\left\|x_{n+1}-w_{n}\right\|^{2} .
$$

Since $\lambda_{n} \rightarrow \lambda>0$, we have $\bar{\kappa}_{n} \rightarrow \frac{1}{2}(1-\mu)$. Thus, it follows from $0<\alpha_{n} \leq \alpha^{*}$ that

$$
\frac{\bar{\kappa}_{n}+1-\alpha_{n}}{\alpha_{n}} \geq \frac{\bar{\kappa}_{n}+1-\alpha^{*}}{\alpha^{*}} \rightarrow \frac{\frac{1}{2}(1-\mu)+1-\alpha^{*}}{\alpha^{*}}=\frac{3-\mu-2 \alpha^{*}}{2 \alpha^{*}}>\bar{\varepsilon} .
$$

Thus, there exists $n_{0} \geq 1$ such that

$$
\frac{\bar{\kappa}_{n}+1-\alpha_{n}}{\alpha_{n}}>\bar{\varepsilon}, \forall n \geq n_{0} .
$$

Combining relations (4.7) and (4.8), we obtain

$$
\left\|x_{n+1}-x^{*}\right\|^{2} \leq\left\|w_{n}-x^{*}\right\|^{2}-\bar{\varepsilon}|| x_{n+1}-w_{n} \|^{2}, \forall n \geq n_{0} .
$$

Set $\bar{\varphi}_{n}:=\left\|x_{n}-x^{*}\right\|^{2}-\theta_{n}\left\|x_{n-1}-x^{*}\right\|^{2}+\theta_{n}(1+\bar{\varepsilon})|| x_{n}-x_{n-1} \|^{2}$ and $\bar{K}:=\bar{\varepsilon}-(2 \bar{\varepsilon}+1) \theta>0$. Using Lemma 3.2, we also obtain

$$
\varphi_{n+1}-\varphi_{n} \leq-\bar{K}|| x_{n+1}-x_{n} \|^{2}, \forall n \geq n_{0} .
$$

The rest of the proof is similar to Theorem 3.1. Theorem 4.1 is proved.

\section{COMPUTATIONAL EXPERIMENTS}

In this section, we consider some examples to illustrate the affect of the inertial term to the numerical behavior of Algorithm 1. We show the behavior of this algorithm because, in the mentioned examples below, the Lipschitz-type constants of the bifunction are known. Note that if $\theta_{n}=0$, then Algorithm 1 becomes the original algorithm, which is without inertial effect. All the programs are written in Matlab 7.0 and computed on a PC Desktop Intel(R) Core(TM) i5-3210M CPU @ 2.50GHz 2.50 GHz, RAM 2.00 GB.

We choose $\lambda_{n}=0.5 \min \left\{\frac{1}{2 c_{1}}, \frac{1}{2 c_{2}}\right\}, \lambda^{*}=0.99 \min \left\{\frac{1}{2 c_{1}}, \frac{1}{2 c_{2}}\right\}$ and

$$
\alpha_{n}=\alpha^{*}:=\frac{1}{2}+\frac{1}{4} \min \left\{1-2 c_{1} \lambda^{*}, 1-2 c_{2} \lambda^{*}\right\} \text {. }
$$

Hence $\varepsilon=1$. Six parameters of $\theta_{n}$ are here chosen to check as

$$
\theta_{n} \in\{0,0.05,0.1,0.2,0.25,0.3\} \text {. }
$$

The starting points are $x_{0}=x_{1}=(1,1, \ldots, 1)^{T} \in \mathfrak{R}^{m}$. The feasible set is a box defined by

$$
C=\left\{x \in \mathfrak{R}^{m}:-5 \leq x_{i} \leq 5, i=1,2, \ldots, m\right\} .
$$

From Algorithm 1, we see that if $y_{n}=w_{n}$ then $y_{n}$ is a solution of the problem. Thus, we use the sequence $D_{n}=\left\|y_{n}-w_{n}\right\|^{2}, n=0,1,2, \ldots$ to study the convergence of the proposed algorithm. The data is generated randomly such that all the conditions of the problem are satisfied.

Example 5.1. Consider a linear bifunction $f: \mathfrak{R}^{m} \times \mathfrak{R}^{m} \rightarrow \mathfrak{R}$ defined by $f(x, y)=\langle P x+Q y+q, y-x\rangle$, where $q \in \mathfrak{R}^{m}$ and $P, Q$ are two $m \times m$ matrices such that $Q$ is symmetric positive semidefinite and $Q-P$ is symmetric negative semidefinite [7]. The bifunction $f$ is pseudomonotone and satisfies the Lipschitz-type condition with $c_{1}=c_{2}=\|P-Q\| / 2$. The numerical behavior of $D_{n}$ is described in Figure 1 and Figure 2 for respectively $m=20$ and $m=50$. 


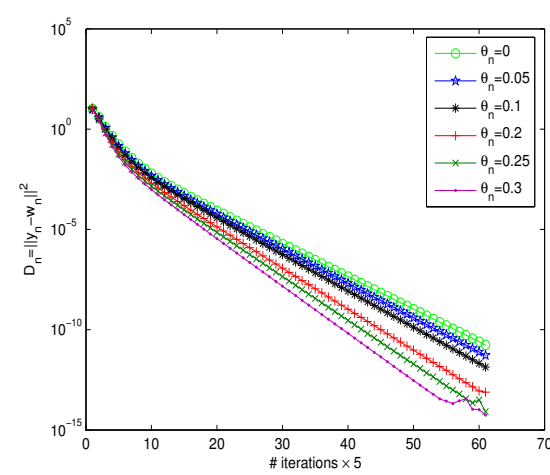

Figure 1. Example 1 for $m=20$.

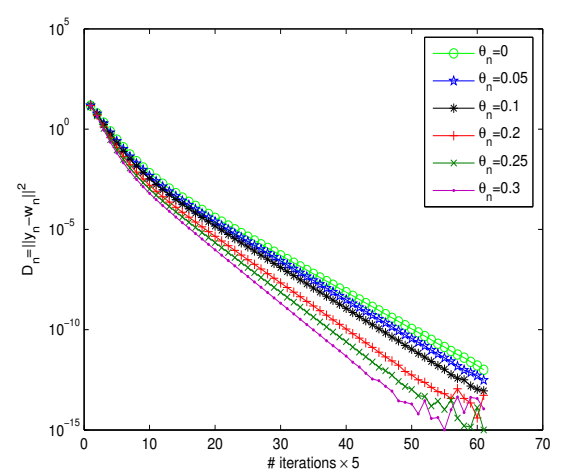

Figure 2. Example 1 for $m=50$.

Example 2. Consider a nonlinear bifunction $f: \mathfrak{R}^{m} \times \mathfrak{R}^{m} \rightarrow \mathfrak{R}$ of the form $f(x, y)=\langle F(x), y-x\rangle$, where $F(x)=A x+P(x)$ and $A$ is a $m \times m$ symmetric semidefinite matrix and $P(x)$ is the proximal mapping of the function $g(x)=\frac{1}{4}\|x\|^{4}$, i.e.,

$$
P(x)=\arg \min \left\{\frac{\|y\|^{4}}{4}+\frac{1}{2}\|y-x\|^{2}: y \in \mathfrak{R}^{m}\right\} .
$$

In this case, $f$ is pseudomonotone and satisfies the Lipschitz-type condition with $c_{1}=c_{2}=$ $\frac{1}{2}(\|A\|+1)$. The numerical results are showed in Figure 3 and Figure 4.

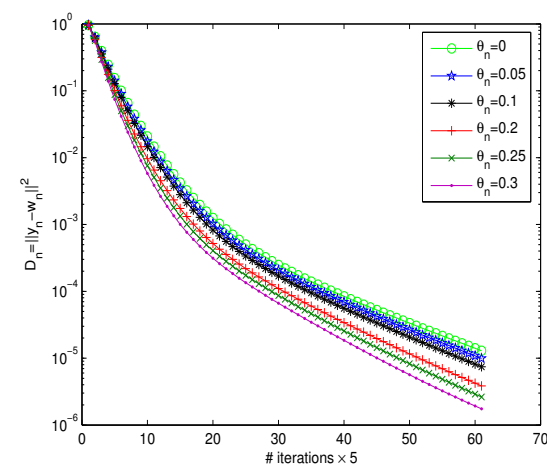

FIGURE 3. Example 2 for $m=20$.

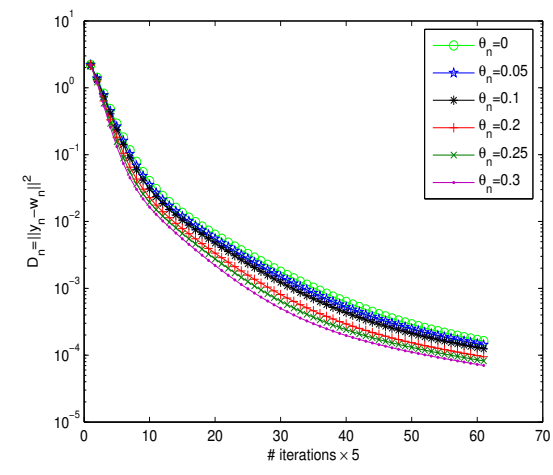

FIGURE 4. Example 2 for $m=50$.

From the aforementioned numerical results, we see that the proposed algorithm with inertial effect $\left(\theta_{n} \neq 0\right)$ seems better than the classical extragradient algorithm $\left(\theta_{n}=0\right)$. Moreover, it is also seen that the larger $\theta_{n}$ is, the better the convergence of the new algorithm is.

\section{Conclusions}

The paper proposed two relaxed inertial extragradient methods for solving the equilibrium problem in Hilbert spaces. The algorithms are constructed around the proximal-like mapping of a bifunction and the inertial method. The algorithms can be implemented with or without 
knowing previously the Lipschitz-type constants of bifunctions. Theorems of weak convergence were established under some mild conditions. Several of numerical results confirmed that the proposed algorithm with inertial effect seems to be better than the original method.

\section{Acknowledgments}

The first author was supported by Vietnam National Foundation for Science and Technology Development (NAFOSTED) under Grant No. 101.01-2020.06.

\section{REFERENCES}

[1] E. Blum, W. Oettli, From optimization and variational inequalities to equilibrium problems, Math. Program. 63 (1994), 123-145.

[2] L.D. Muu, W. Oettli, Convergence of an adative penalty scheme for finding constrained equilibria, Nonlinear Anal. 18 (1992), 1159-1166.

[3] K. Fan, A minimax inequality and applications, In: Shisha, O. (ed.) Inequality III, 103-113. Academic Press, New York, 1972.

[4] S.D. Flam, A.S. Antipin, Equilibrium programming and proximal-like algorithms, Math. Program. 78 (1997), 29-41.

[5] D.V. Hieu, L.D. Muu, P.K. Anh, Parallel hybrid extragradient methods for pseudomonotone equilibrium problems and nonexpansive mappings, Numer. Algo. 73 (2016), 197-217.

[6] D.V. Hieu, L.D. Muu, P.K. Anh, Modified hybrid projection methods for finding common solutions to variational inequality problems, Comput. Optim. Appl. 66 (2017), 75-96.

[7] D.V. Hieu, Parallel extragradient-proximal methods for split equilibrium problems, Math. Model. Anal. 21 (2016), 478-501.

[8] D.V. Hieu, New subgradient extragradient methods for common solutions to equilibrium problems, Comput. Optim. Appl. 67 (2017), 571-594.

[9] D.V. Hieu, P.K. Quy, L.V. Vy, Explicit iterative algorithms for solving equilibrium problems, Calcolo 56 (2019), 11.

[10] D.V. Hieu, B.H. Thai, P. Kumam, Parallel modified methods for pseudomonotone equilibrium problems and fixed point problems for quasinonexpansive mappings, Adv. Oper. Theory. 5 (2020), 1684-1717.

[11] D.V. Hieu, P.K. Quy, L.T. Hong, L.V. Vy, Accelerated hybrid methods for solving pseudomonotone equilibrium problems, Adv. Comput. Math. 58 (2020), 46.

[12] D.V. Hieu, J.J. Strodiot, L.D. Muu, Strongly convergent algorithms by using new adaptive regularization parameter for equilibrium problems. J. Comput. Appl. Math. 376 (2020) 112844.

[13] G. Mastroeni, Gap function for equilibrium problems, J. Global Optim. 27 (2003), 411-426.

[14] A. Moudafi, Proximal point algorithm extended to equilibrum problem, J. Nat. Geometry 15 (1999), 91-100.

[15] T.D. Quoc, L.D. Muu, V.H. Nguyen, Extragradient algorithms extended to equilibrium problems, Optimization 57 (2008), 749-776.

[16] P.T. Vuong, J.J. Strodiot, V.H. Nguyen, Extragradient methods and linesearch algorithms for solving Ky Fan inequalities and fixed point problems, J. Optim. Theory Appl. 155 (2012), 605-627.

[17] B. Martinet, Régularisation d' inéquations variationelles par approximations successives, Rev. Fr. Autom. Inform. Rech. Opér., Anal. Numér. 4 (1970), 154-159.

[18] R.T. Rockafellar, Monotone operators and the proximal point algorithm, SIAM J. Control Optim. 14 (1976), 877-898.

[19] I.V. Konnov, Application of the proximal point method to nonmonotone equilibrium problems, J. Optim. Theory Appl. 119 (2003), 317-333.

[20] B.T. Polyak, Some methods of speeding up the convergence of iterative methods, Zh. Vychisl. Mat. Mat. Fiz. 4 (1964), 1-17.

[21] F. Alvarez, H. Attouch., An inertial proximal method for maximal monotone operators via discretization of a nonlinear oscillator with damping, Set-Valued Anal. 9 (2001), 3-11. 
[22] F. Alvarez, Weak convergence of a relaxed and inertial hybrid projection-proximal point algorithm for maximal monotone operators in Hilbert space, SIAM J. Optim. 14 (2004), 773-782.

[23] R.I. Bot, E.R. Csetnek, C. Hendrich, Inertial Douglas-Rachford splitting for monotone inclusion problems, Appl. Math. Comput. 256 (2015), 472-487.

[24] A. Beck, M. Teboulle, A fast iterative shrinkage-thresholding algorithm for linear inverse problems, SIAM J. Imaging Sci. 2 (2009), 183-202.

[25] R.I. Bot, E.R. Csetnek, An inertial alternating direction method of multipliers, Minimax Theory Appl. 1 (2016), 29-49.

[26] R.I. Bot, E.R. Csetnek, A hybrid proximal-extragradient algorithm with inertial effects, Numer. Funct. Anal. Optim. 36 (2015), 951-963.

[27] R.I. Bot, E.R. Csetnek, S.C. Laszlo, An inertial forward-backward algorithm for the minimization of the sum of two nonconvex functions, EURO J. Comput. Optim. 4 (2016), 3-25.

[28] R.I. Bot, E.R. Csetnek, An inertial Tseng's type proximal algorithm for nonsmooth and nonconvex optimization problems, J. Optim. Theory Appl. 171 (2016), 600-616.

[29] R.I. Bot, E.R. Csetnek, An inertial forward-backward-forward primal-dual splitting algorithm for solving monotone inclusion problems. Numer. Algor. 71 (2016), 519-540.

[30] C. Chen, S. Ma, J. Yang, A general inertial proximal point algorithm for mixed variational inequality problem, SIAM J. Optim. 25 (2015), 2120-2142.

[31] P. Ochs, Y. Chen, T. Brox, T. Pock, iPiano: inertial proximal algorithm for non-convex optimization, SIAM J. Imaging Sci. 7 (2014), 1388-1419.

[32] D.A. Lorenz, T. Pock, An inertial forward-backward algorithm for monotone inclusions, J. Math. Imaging Vis. 51 (2015), 311-325.

[33] A. Moudafi, Second-order differential proximal methods for equilibrium problems, J. Inequal. Pure and Appl. Math. 4 (2003) Art. 18.

[34] Z. Chbani, H. Riahi, Weak and strong convergence of an inertial proximal method for solving Ky Fan minimax inequalities, Optim. Lett. 7 (2013), 185-206.

[35] D.V. Hieu, An inertial-like proximal algorithm for equilibrium problems, Math. Meth. Oper. Res. 88 (2018), 399-415.

[36] D.V. Hieu, A. Gibali, Strong convergence of inertial algorithms for solving equilibrium problems, Optim. Lett. 14 (2020), 1817-1843.

[37] G.M. Korpelevich, The extragradient method for finding saddle points and other problems, Ekonomika i Matematicheskie Metody 12 (1976), 747-756.

[38] D.V. Hieu, Y.J. Cho, Y-B. Xiao, Modified extragradient algorithms for solving equilibrium problems, Optimization 67 (2018), 2003-2029.

[39] H.H. Bauschke, P.L. Combettes, Convex Analysis and Monotone Operator Theory in Hilbert Spaces, Springer, New York, 2011. 